\title{
Culture-negative endocarditis due to Bartonella quintana
}

\author{
V M Moodley, M T S Zeeman, C H J van Greune, C Corcoran
}

Dr Mischka Moodley is a consultant medical microbiologist at Ampath National Laboratory Services, based in Cape Town, South Africa. Dr Tienie Zeeman is a consultant physician at Netcare N1 City Hospital in Cape Town. Dr Johan van Greune is a consultant clinical pathologist at Ampath National Laboratory Services in Cape Town. Dr Craig Corcoran is a consultant clinical virologist at the Molecular Biology Unit, Ampath National Reference Laboratory, Pretoria, South Africa.

Corresponding author: VM Moodley (moodleym@ampath.co.za)

Bartonella spp. was first described as a possible cause of culture-negative endocarditis in 1993, and has since emerged as a significant cause of this condition worldwide. We describe a complicated case of culture-negative endocarditis in an immune-competent male patient, which was confirmed on resected heart valves to have been caused by Bartonella quintana by broad-range $16 \mathrm{~S}$ ribosomal RNA polymerase chain reaction. The objective of this report is to highlight the clinical, diagnostic and therapeutic challenges of Bartonella endocarditis.

S Afr Med J 2016;106(5):470-471. DOI:10.7196/SAMJ.2016.v106i5.9062

\section{Case report}

A 30-year-old man presented to his physician in October 2013 with a 6-week history of progressive dyspnoea, chest pain and non-productive cough. Of note, he had no history of congenital or rheumatic heart disease, was not known to be HIV-infected or immunocompromised from another medical condition, and was employed in the insurance industry. There was no significant history of travel or contact with domestic animals. Furthermore, he denied any intravenous drug use.

Physical examination revealed that he was pyrexial, with a pulse rate of 116 beats/minute and a blood pressure of 120/66 mmHg. He had a grade 4/6 mitral regurgitation murmur and a grade $1 / 4$ aortic regurgitation murmur. There was no clinical evidence of microembolic phenomena. His spleen was just palpable under the costal margin. Chest radiography revealed biventricular cardiomegaly with upper lobe diversion, consistent with cardiac decompensation. An echocardiogram demonstrated vegetations of both the aortic and mitral valves, with incompetency across both valves. An ultrasound scan of the abdomen showed small bilateral pleural effusions, hepatosplenomegaly and a wedge-shaped zone of splenic infarction.

Laboratory investigations revealed a haemoglobin of $9.5 \mathrm{~g} / \mathrm{dL}$, leucocyte count $8.63 \times 10^{9} / \mathrm{L}$, platelets $245 \times 10^{9} / \mathrm{L}$, urea $10.8 \mathrm{mmol} / \mathrm{L}$, creatinine $96 \mu \mathrm{mol} / \mathrm{L}$ and C-reactive protein (CRP) $42 \mathrm{mg} / \mathrm{L}$. Urinalysis revealed $>100$ erythrocytes per high-power field and no casts. Four sets of blood cultures were performed, and the patient was commenced on penicillin and gentamicin for suspected native-valve endocarditis. A further three sets of blood cultures were done the following day. Rheumatoid factor was $393 \mathrm{IU} / \mathrm{mL}$ with negative antinuclear antibodies, antibodies to extractable nuclear antigen, and antibodies to cyclic citrullinated peptide. Decreased complement C3 and C4 of $0.43 \mathrm{~g} / \mathrm{L}$ and $0.07 \mathrm{~g} / \mathrm{L}$, respectively, were also noted. The blood cultures remained negative, and although the patient was stable he continued to have temperature spikes while the CRP remained largely unchanged.

Serological investigations for the common causes of culture-negative endocarditis were performed (Table 1). Brucella polymerase chain reaction (PCR) on blood was negative. In the interim, the penicillin was substituted with teicoplanin and the gentamicin was continued. As the patient developed a degree of renal impairment, the gentamicin was discontinued and meropenem was commenced. Finally, in view
Table 1. Serological investigations for the aetiology of culturenegative endocarditis

\begin{tabular}{|c|c|}
\hline Organism & Result \\
\hline Mycoplasma pneumoniae & IgM-; IgG+ \\
\hline Legionella pneumophila & $\operatorname{IgM}+(1: 100) ; \operatorname{IgG}+(1: 200)$ \\
\hline Chlamydophila psittacci & IgA-; IgM-; IgG+ \\
\hline Chlamydia trachomatis & IgA-; IgM-; IgG+ \\
\hline Bartonella henselae & IgM+; IgG+ (1:512) \\
\hline Bartonella quintana & $\operatorname{IgM}+; \operatorname{IgG}+(1: 512)$ \\
\hline Brucella spp. & IgM-; IgG+ \\
\hline Coxiella burnetii & IgM-; IgGp1-; IgGp2+ (1:128) \\
\hline
\end{tabular}

of the serological results and negative blood cultures, doxycycline and rifampicin were also added as per international endocarditis guidelines. ${ }^{[1]}$ After the addition of the latter two antibiotics, the patient's general condition improved and his temperature spikes dissipated.

The patient was taken for valve-replacement surgery approximately 4 weeks later. The resected mitral and aortic valves were sent for microbiological culture as well as broad-range 16S ribosomal RNA (rRNA) PCR. Histological examination confirmed fibrinous vegetations with associated neutrophils, lymphocytes and histiocytes. No pathogens were detected with periodic acid-Schiff or Brown-Hopps stains. Extended microbiological culture did not yield any atypical bacteria. A $16 \mathrm{~S}$ rRNA PCR was performed and the PCR products sequenced, which confirmed the presence of Bartonella quintana DNA in the resected aortic heart valve. After surgery, the patient was continued on doxycycline $100 \mathrm{mg} 12$-hourly for 3 months. He remains well on subsequent follow-up examinations.

\section{Discussion}

Bartonella spp. was first discovered in 1909 by A L Barton and described as a cause of culture-negative endocarditis in 1993. ${ }^{[2]}$ Bartonellae are fastidious, oxidase-negative, pleomorphic Gram-negative bacteria. Their growth is haemin-dependent; therefore, they only grow on 
enriched media that contain haemin-rich rabbit or horse blood. Routine culture protocols do not detect these organisms, and prolonged culture (up to 45 days) is required.

$B$. henselae is the aetiological agent of cat-scratch disease, bacillary angiomatosis, meningo-encephalitis and chronic bacteraemia. The domestic cat and its fleas (Ctenocephalides felis) are the associated reservoir and vector for the organism, respectively. In contrast, $B$. quintana is the cause of trench fever, an infection that resulted in significant mortality during World War I and II. This organism is transmitted by the human body louse (Pediculus humanus) and is responsible for infections in refugees and the homeless. B. quintana is also associated with bacillary angiomatosis, particularly in HIVinfected individuals and cases of chronic bacteraemia. A study in South Africa (SA), conducted between 2007 and 2009, revealed a high PCR prevalence of Bartonella DNA in blood specimens: $22.5 \%(n=382)$ in HIV-infected patients, 9.5\% $(n=42)$ in healthy volunteers, $23.5 \%(n=98)$ in domestic cats, $9 \%(n=179)$ in domestic dogs, and 25\% $(n=124)$ in rodent samples. ${ }^{[3]}$ Frean et al. ${ }^{[4]}$ found that $10 \%(n=188)$ of patients who presented to an outpatient HIV clinic in Johannesburg had a Bartonella bacteraemia detected by nested PCR performed on blood.

Bartonellae are highly adapted to their mammalian hosts and can persist in the bloodstream owing to intra-erythrocytic parasitism. This bacterium has several virulence attributes, including a type IV secretion system that delivers effector molecules that subvert host cells, induction of angiogenesis by stimulating apoptosis and activation of hypoxia-inducing factor 1 , adhesins and lipopolysaccharides in its outer membrane. ${ }^{[5]}$

The first published case of Bartonella endocarditis in SA was reported in $1996 .{ }^{[6]}$ Six species have been implicated in infective endocarditis, i.e. B. quintana, B. henselae, B. elizabethae, B. vinsonii, B. koehlerae, and B. alsatica. The majority of cases (95\%) are caused by $B$. quintana $(75 \%)$ and $B$. henselae $(25 \%) .{ }^{[6]}$

Risk factors for Bartonella endocarditis include homelessness, alcoholism, infestation with body lice (B. quintana), contact with cats and pre-existing valvular disease (B. henselae). ${ }^{[7]}$

Raoult et al. ${ }^{[6]}$ who described a case series of 22 patients with Bartonella endocarditis, reported that the clinical features of the disease were similar to those of patients with endocarditis caused by other organisms (Duke critera), except that patients were likely to be male and had undergone valvular surgery. Moreover, only $25 \%$ of their patients with confirmed Bartonella endocarditis had positive blood cultures. There appears to be an unknown predilection to infect the aortic valve. Although it is generally associated with native-valve endocarditis, there are reports of aggressive prosthetic valve endocarditis. ${ }^{[8]}$ The echocardiograms of the majority of patients diagnosed with $B$. henselae endocarditis are consistent with endocarditis.

Serological tests often give an indication of the likely pathogen in culture-negative endocarditis. Our case was complicated by positive acute serology for both Legionella spp. and Coxiella burnetii (phase 2 serology indicates a recent infection), which also cause culture-negative endocarditis. Serological cross-reactions with Chlamydophila spp. and Coxiella spp. (Q-fever) have also been reported. ${ }^{[6,9]}$ Graham et al. ${ }^{[10]}$ reported a case of prosthetic valve endocarditis due to Q-fever, showing seropositivity to a broad range of pathogens, including B. quintana, B. henselae, C. burnetii, Rickettsia rickettsii and Ehrlichia chaffeensis. Similar to our case, this case highlights the difficulties of making a diagnosis based on serological testing in the absence of positive microbiological culture.

The raised rheumatoid factor alerted us to the possibility of a noninfective cause of endocarditis, particularly neoplastic or autoimmune. However, the negative antinuclear antibodies and histological findings did not support this diagnosis. Interestingly, Fournier et al. ${ }^{[1]}$ identified $19(2.5 \%)$ of 819 endocarditis cases that had non-infectious causes, such as Libmann-Sacks endocarditis. This is an important consideration in culture-negative endocarditis cases. Of note, Bojalil et al. ${ }^{[12]}$ found that $68 \%(n=18)$ of their cohort with endocarditis had a positive rheumatoid factor. Other highly specific antibodies were absent. These findings are similar to those in our case.

Histological examination of a resected valve with Bartonella infection often reveals inflamed connective tissue with focal granulation tissue. Warthin-Starry silver staining may reveal small, dark-staining aggregates of bacteria, but these are not specific for bartonellae. Broad-range bacterial PCR-based testing of resected cardiac valves that target the 16S rRNA gene has played an important role in the diagnosis of Bartonella endocarditis and other causes of culture-negative endocarditis. ${ }^{[13]}$ PCR on excised heart valves is considered a sensitive and reliable method. ${ }^{[14]}$ False-negative results of PCR on resected valves can occur owing to the presence of PCR inhibitors, sequence variability underlying primers, or the presence of bacterial DNA in quantities less than the limit of detection of the assay. False-positives may occur where the valve is contaminated during surgical removal or transport and processing in the laboratory. It is therefore important to correlate a positive $16 \mathrm{~S}$ rRNA PCR and sequence with the organism that has been isolated and the clinical features of the patient. In addition to the patient described in this case report, our laboratory has diagnosed several cases of $B$. quintana endocarditis and other causes of endocarditis using a broad-range $16 \mathrm{~S}$ rRNA PCR on excised heart valves.

The optimal therapy for Bartonella endocarditis is unknown. Generally, the tetracyclines (doxycycline) or a beta lactam is combined with a bactericidal agent, such as gentamicin, which is added for the initial $2-4$ weeks of therapy. ${ }^{[1,15]}$ If a patient does not tolerate gentamicin, rifampicin may be used as an alternative. ${ }^{[15]}$ Most guidelines advise 6 weeks of therapy, although some authors suggest extending therapy to 3 - 6 months due to the severity of the illness, low cost of the treatment and lack of treatment duration data. ${ }^{[16]}$ Many patients will, however, require valve replacement. ${ }^{[7]}$

Our report highlights the importance of Bartonella spp. as a cause of culture-negative infectious endocarditis and the role of broad-range bacterial molecular diagnostics as a promising tool in determining the aetiology of endocarditis as well as the difficulties in laboratory diagnosis and management of this condition. endocarditis in adults: A report of the Working Party of the British Society for Antimicrobial Chemotherapy. J Antimicrob Chemother 2012;67(2):269-289. DOI:10.1093/jac/dkr450

2. Spach DH, Callis KP, Paauw DS, et al. Endocarditis caused by Rochalimaea quintana in a patient infected with human immunodeficiency virus. J Clin Microbiol 1993;31(3):692.

3. Trataris AN, Rossouw J, Arntzen L, Karstaedt A, Frean J. Bartonella spp. in human and animal populations in Gauteng, South Africa, from 2007 to 2009. Onderstepoort J Vet Res 2012;79(2):452. DOI:10.4102/ojvr.v79i2.452

4. Frean J, Arndt S, Spencer D. High rate of Bartonella henselae infection in HIV-positive outpatients in Johannesburg,

South Africa. Trans R Soc Trop Med Hyg 2002;96(5):549-550. DOI:10.1016/S0035-9203(02)90437-2
5. Minnick MF, Battisti JM. Pestilence, persistence and pathogenicity: Infection strategies of Bartonella. Minnick MF, Battisti JM. Pestilence, persistence and pathog

Future Microbiol 2009;4(6):743-58. DOI:10.2217/fmb.09.41
6. Raoult D, Fournier PE, Drancourt M, et al. Diagnosis of 22 cases of Bartonella endocarditis. Ann Intern 6. Raoult D, Fournier PE, Drancourt M, et al. Diagnosis of 22 cases of Bartonella
Med 1996;125:646-652. DOI:10.7326/0003-4819-125-8-199610150-00004

Med 1996;125:646-652. DOI:10.7326/0003-4819-125-8-199610150-00004
7. Raoult D, Fournier PE, Vandenesch F, et al. Outcome and treatment of Bartonella endocarditis. Arch 7. Raoult D, Fournier PE, Vandenesch F, et al. Outcome and treatment
Intern Med 2003;163(2):226-230. DOI:10.1001/archinte.163.2.226

8. Hajj-Chahine J, Houmaida H, Plouzeau C, Tomasi J, Corbi P. Bartonella as a cause of mechanical prosthetic aortic root abscess. Ann Thorac Surg 2012;93:e93-e95. DOI:10.1016/j.athoracsur.2011.11.051 9. La Scola B, Raoult D. Serological cross-reactions between Bartonella quintana, Bartonella henselae, and Coxiella burnetii. J Clin Microbiol 1996;34(9):2270-2274.

10. Graham JV, Baden L, Tsiodras S, Karchmer AW. Q fever endocarditis associated with extensive serological cross-reactivity. Clin Infect Dis 2000;30(3):609-610. DOI:10.1086/313701

11. Fournier PE, ThunyF, Richet H, et al.Comprehensive diagnostic strategy for blood culture-negative endocarditis: A prospective study of 819 new cases. Clin Infect Dis 2010;51(2):131-140. DOI:10.1086/653675

12. Bojalil R, Mazón-González B, Carrillo-Córdova JR, Springall R, Amezcua-Guerra LM. Frequency and clinical significance of a variety of autoantibodies in patients with definite infective endocarditis. J Clin clinical significance of a variety of autoantibodies in patients with defin
Rheumatol 2012;18(2):67-70. DOI:10.1097/RHU.0b013e318247 cafo

13. Breitkopf C, Hammel D, Scheld HH, Peters G, Becker K. Impact of a molecular approach to improve the microbiological diagnosis of infective heart valve endocarditis. Circulation 2005;111(11):1415-
the the microbiological diagnosis of infective heart val
1421. DOI:10.1161/01.CIR.0000158481.07569.8D

14. Fournier PE, Lelievre H, Eykyn SJ, et al. Epidemiologic and clinical characteristics of Bartonella quintana and Bartonella henselae endocarditis: A study of 48 patients. Medicine (Baltimore) 2001;80(4):245-251. 15. Rolain JM, Brouqui P, Koehler JE, Maguina C, Dolan MJ, Raoult D. Recommendations for treatment of human infections caused by Bartonella species. Antimicrob Agents Chemother 2004;48(6):1921-1933. 16. Ohl ME, Spach DH. Bartonella quintana and urban trench fever. Clin Infect Dis 2000;31(1):131-135. DOI:10.1086/313890

Accepted 19 January 2015. 